\title{
Hazte un cell-fie
}

Carlota Gómez ${ }^{\mathrm{a}^{*}}$, Elisa Langa ${ }^{\mathrm{a}}$, Jose Juan Verón ${ }^{\mathrm{c}}$; Marta Uriel ${ }^{\mathrm{a}}$, Desirée Acebes ${ }^{\mathrm{b}}$; Monika Woźniak ${ }^{\mathbf{b}}$, Eva $\mathbf{M}^{\mathbf{a}}$ Terrado.

${ }^{a}$ Facultad de Ciencias de la Salud, ' ${ }^{b}$ Instituto de Lenguas Modernas; 'Facultad de Comunicación y Ciencias Sociales. Universidad San Jorge, Zaragoza, España * cgomez@usj.es

\begin{abstract}
The objective of this project is to develop inclusive scientific workshops which combine promoting "scientific literacy" in primary schools and addressing the training needs of university students through the Service-Learning methodology.

The project involved the participation of 50 students of Pharmacy ( $1^{\text {st }}$ year) and Primary Education (3 ${ }^{\text {rd }}$ year) at San Jorge University, along with 6 primary school teachers and 75 primary schoolchildren (2 ${ }^{\text {nd }}$ year) from various schools in Zaragoza. The project was divided into four phases. The first one focused on the search of specific information by all students involved and the development of learning materials to organize all the content related to cells. The second phase took the form of a cooperative seminar for students of both degree programmes. During the seminar, Education students received specific training (which allowed them to clarify and expand their knowledge about cell biology) and taught Pharmacy students how to adapt scientific language to a non-specialized audience (didactic transposition). The third phase was dedicated to creating informative materials (videotutorial recording, construction of cell models and design of a microscopy workshop for children). As a fourth phase of the project, a series of practical workshops were held with different groups of primary schoolchildren, using the materials developed in the previous phase. The activity was very highly rated by both Pharmacy and Education students whereas the workshops had a very positive impact on schoolchildren. In this respect, $98.5 \%$ of the children liked the activity and 93\% indicated that they had learned things they did not know about cells.
\end{abstract}

Keywords: Teaching innovation, service-learning, scientific literacy, curricular integration, dissemination, didactic transposition.

\section{Resumen}

Este proyecto tiene como objetivo desarrollar talleres cientificos inclusivos destinados a promover la "alfabetización científica" en centros de Educación 
Primaria a la vez que se satisfacen las necesidades formativas de estudiantes universitarios a través de la metodología de Aprendizaje-Servicio.

En el proyecto han participado 50 estudiantes de $1^{\circ}$ del grado en Farmacia y de $3^{\circ}$ del grado en Educación Primaria de la Universidad San Jorge, junto con 6 maestros y 75 estudiantes de $2^{\circ}$ de Educación Primaria en varios colegios de Zaragoza. El proyecto se estructuró en cuatro fases. La primera se concretó en la búsqueda de información específica por parte de los estudiantes de ambos grados y el desarrollo de materiales de aprendizaje para organizar toda los contenidos relativos a las células. La segunda fase se materializó en un seminario cooperativo entre discentes de ambos grados en el cual los estudiantes de educación recibieron formación especifica (que les permitió aclarar y ampliar sus conocimientos sobre biología celular) a la vez que enseñaron a los estudiantes de farmacia a adaptar el lenguaje científico para un público no especializado (transposición didáctica). La tercera fase se enfocó en la creación de materiales divulgativos (grabación de videotutoriales, construcción de maquetas celulares y diseño de un taller de microscopía para niños). Como cuarta fase del proyecto, se llevaron a cabo una serie de talleres prácticos con diversos grupos de estudiantes de $2^{\circ}$ de Educación Primaria, utilizando los materiales desarrollados en la fase anterior.Tanto los estudiantes del grado en Farmacia como los estudiantes del Grado en Educación valoraron de forma muy positiva la actividad. Por otro lado, los talleres realizados tuvieron un impacto muy positivo en los escolares asi un 98,5\% de los mismos indicó que la actividad les habia gustado mucho y un 93\% que habian aprendido cosas que no sabian sobre las células.

Palabras clave: Innovación docente, aprendizaje-Servicio, alfabetización científica, integración curricular, divulgación, transposición didáctica.

\section{Introducción}

En una sociedad altamente tecnificada como la actual, la alfabetización científica resulta imprescindible para contribuir a formar ciudadanos cuya formación les permita reflexionar y tomar decisiones apropiadas en temas relacionados con la ciencia y la tecnología (Solbes y Vilches, 1997). Por ello, desde los diversos niveles educativos se debe tener muy presente, y desde un enfoque pedagógico y divulgativo, la necesidad de promover e incentivar la "alfabetización científica" (UNESCO-ICSU, 1999a-b), interpretada en su triple dimensión cognitiva (saber ciencia), procedimental (hacer ciencia) y actitudinal (valorar la ciencia). Por otro lado, la idea de "ciencia para todas las personas", significa una enseñanza de la misma que no excluya a nadie, y que esté íntimamente asociada a los principios educativos de comprensividad y equidad. Esta premisa se vincula también con hacer más accesible, interesante y significativa la "ciencia". La enseñanza de la ciencia es un instrumento de valor inestimable para despertar la curiosidad y espíritu crítico, fomentar la creatividad y desarrollar destrezas y actitudes, actividades esenciales para desarrollarlo desde el punto de vista psíquico (Sabariego y Manzanares, 2006). Desgraciadamente, la ausencia de formación científica básica es un hecho generalizado en nuestra sociedad como demuestran los 
resultados obtenidos en una encuesta realizada por la Fundación Española para la Ciencia y la Tecnología en el 2008. Esta investigación puso de manifiesto que el 45,8 \% de los encuestados consideraban que su educación científica había sido baja o muy baja. Esta falta de formación dificulta la asimilación por parte de la ciudadanía de los conocimientos científicos haciendo que esta no tenga correspondencia con el crecimiento exponencial de estos provocando un enorme desfase entre los descubrimientos y su vertido a la cultura popular. Por ello, la brecha del conocimiento que se está generando entre los individuos que lo generan y aquellos a los que van dirigidas las aplicaciones provenientes de esos conocimientos es cada vez mayor.

El poder que los medios de comunicación ejercen en la toma de decisiones de los ciudadanos en la sociedad actual resulta evidente. Una información incorrecta bien por falta de rigor o de forma intencionada (las controvertidas fake news) generan un impacto altamente negativo en la sociedad. En el ámbito del periodismo científico y médico, este impacto puede tener consecuencias graves para la salud del lector crédulo. Por ello el futuro periodista debe tomar conciencia de la importancia de su papel en la alfabetización científica de la sociedad. Así, deberá conocer los fundamentos de la información científica y del periodismo especializado para aplicarlos en la elaboración de contenidos veraces.

Ya desde edad escolar, el acceso de los ciudadanos a la ciencia es limitado. Una de las causas radica posiblemente en la creencia entre docentes, padres y responsables de los centros educativos de que realizar experimentos científicos en el ámbito escolar es caro y complejo. Este hecho junto con la escasa formación científica recibida por los maestros de educación primaria desanima a los docentes y evita que los centros inviertan recursos en la adquisición de materiales y/o equipos destinados a tal fin. Si bien en el caso de la enseñanza secundaria los docentes de las asignaturas de ciencias poseen formación científica, la falta de medios materiales y personales dificulta en ocasiones la implementación real de ciencia práctica en las aulas. En el ámbito Universitario la situación no es diferente y salvo contadas excepciones no existen programas estables de divulgación científica en Facultades en las que se imparten estudios no relacionados con las ciencias experimentales. Por ello, la formación científica de los futuros maestros permitiría contribuir de forma altamente significativa a la alfabetización científica de la sociedad. El maestro científicamente capacitado sabrá imprimir en sus alumnos la curiosidad y afán de conocimiento propios del pensamiento científico. Además, los estudiantes que reciban esta formación actuarán a su vez como "pequeños divulgadores" provocando un impacto positivo en su entorno cercano.

El farmacéutico constituye un profesional sanitario fácilmente accesible para la población general por ello, debe contribuir a la educación sanitaria de la sociedad. Así desde la denominada atención farmacéutica puede aumentar el conocimiento de la población sobre sus patologías y medicamentos, potenciando la autonomía del paciente a través del consejo farmacéutico. Su completa formación científica además, convierte al farmacéutico en un potencial divulgador cuyo trabajo puede contribuir a mejorar la cultura científica de la sociedad. Por este motivo, el estudiante de farmacia deberá ser capaz de utilizar un lenguaje adecuado con el fin de poder trasmitir sus conocimientos a pacientes de nivel cultural muy diverso. 
La divulgación contribuye a la rendición de cuentas que la investigación debe a la sociedad que la sustenta. El papel de las Universidades en la divulgación científica es fundamental, puesto que una de las misiones de la Universidad, además de la docencia y la investigación es la transferencia del conocimiento a la sociedad. Por tanto, debería existir un doble compromiso de las universidades en relación con la divulgación: Difundir entre la sociedad los resultados de su investigación y formar a sus alumnos como futuros divulgadores de la investigación.

En el contexto teórico de las ciencias de la vida, la teoría celular constituye un pilar fundamental. Esta teoría postula que la célula es la unidad básica de la vida y que todos los seres vivos están constituidos por ellas. La adecuada asimilación de este concepto es imprescindible para una correcta comprensión de numerosos aspectos relacionados con la salud, el medio ambiente o la tecnología. El hecho de que todo nuestro cuerpo esté formado por unidades básicas invisibles al ojo humano resulta cuando menos mágico y poco asequible para la personas no ilustradas en ciencias de la vida. Sin embargo, esta aparente inaccesibilidad al concepto célula resulta fácilmente superable cuando se tiene acceso a un microscopio. Pocas herramientas didácticas pueden ayudar a comprender mejor a niños o adultos la teoría celular que la visualización de sus propias células. Entre la infinidad de "maquinaria" científica disponible pocos aparatos ofrecen tanta versatilidad como el microscopio. Gracias a él se pueden realizar infinidad de experimentos científicos sencillos no solo de biología también de física, de química o de arte. Además, pocas cosas son tan mágicas como esa realidad paralela y diminuta que se muestra bajo sus objetivos. A pesar de ello, el microscopio óptico sencillo, no es un elemento habitual en las aulas. Así lo demuestra una experiencia que año tras año se realiza en el aula de Fundamentos de Biología en primer curso del en farmacia. Cuando se pide a los estudiantes que imaginen una célula, el $95 \%$ de ellos visualizan el dibujo de una célula animal observado en un libro de texto. Es decir, una idea descontextualizada y alejada del concepto de célula como unidad básica de la vida. Así, se detectó que numerosos estudiantes del grado en Farmacia no habían utilizado nunca a lo largo de su formación académica un microscopio. Por ello se decidió realizar un sondeo informal entre diferentes colegios de la ciudad con el fin de determinar el uso de los mismos durante alguna de las etapas formativas. El resultado puso de manifiesto que menos de un $10 \%$ de los centros dispone de microscopios en sus instalaciones. Además, muchos de estos centros tampoco disponen de un espacio destinado a desarrollar talleres científicos. Conscientes de esta necesidad, desde la Universidad San Jorge se planteó el proyecto de Aprendizaje Servicio "Conoce tus células" que gracias a la financiación de Ibercaja permitió llevar a 75 escolares la magia del mundo microscópico junto con estudiantes de los grados de Farmacia y Educación primaria. El fuerte impacto positivo en los aprendizajes de escolares, estudiantes de grado y docentes permite concluir que la metodología utilizada en el presente trabajo contribuirá a sentar las bases para que la Universidad afronte el reto de formar profesionales altamente capacitados que a través de sus áreas de conocimiento contribuyan a la formación científica de nuestra sociedad.

\section{Objetivos}


El presente proyecto tiene como objetivo principal desarrollar y evaluar un proyecto educativo innovador que se centra en realizar una serie de talleres científicos inclusivos destinados a promover e incentivar la "alfabetización científica" en los centros de Educación Primaria (maestros y estudiantes) al mismo tiempo que se satisfacen las necesidades formativas (cognitivas, procedimentales y actitudinales) de los discentes universitarios de los grados en Farmacia y Educación Primaria de la universidad San Jorge a través de un enfoque integrado de aprendizaje formal y servicio a la comunidad.

La consecución del citado objetivo general se abordó a través de los siguientes hitos u objetivos secundarios:

Hito 1: Favorecer la adquisición de competencias profesionales en los estudiantes de Educación Primaria y Farmacia a través de la transposición didáctica con estudiantes y docentes de otras disciplinas.

Hito 2: Contribuir a la formación científica básica de los estudiantes de Educación para favorecer a la implementación futura de la ciencia práctica en el contexto escolar.

Hito 4: Mejorar la capacidad del estudiante de Farmacia para comunicarse y colaborar con el paciente utilizando un lenguaje adecuado con el fin de poder trasmitir sus conocimientos en materia de salud a la sociedad.

Hito 5: Llevar a cabo talleres científicos que contribuyan a la generación de cultura y vocación científica en niños de segundo de educación primaria.

\section{Desarrollo de la innovación}

La enseñanza a otros de los aprendizajes adquiridos está considerada la forma más eficiente de formar profesionales altamente cualificados. Así, la búsqueda de metodologías que permitan un aprendizaje efectivo resulta fundamental en la actual práctica docente (Abadía et al, 2011). Por ello, el planteamiento metodológico de este proyecto se ha realizado acorde con un enfoque centrado en las metodologías activas.

El uso de metodologías participativas desarrolladas en grupos potencia la integración de los conocimientos adquiridos (Walters, 1999; Waters, J et al., 2005). Por otro lado, las actividades incluidas en las denominadas pedagogías de la experiencia buscan favorecer el contacto directo del estudiante con la realidad que debe conocer, desencadenando procesos de aprendizaje significativo (Puig, et al., 2006). Entre las diversas metodologías utilizadas en este ámbito, destaca el Aprendizaje-Servicio (ApS) que combina el servicio a la comunidad con la instrucción académica. El ApS tiene como pilares fundamentales la experiencia, la reflexión y la reciprocidad para favorecer además de la adquisición de conocimientos y competencias profesionales, la responsabilidad social y cívica en el estudiante (Eyler y Giles, 1999). El ApS es una práctica pedagógica con una enorme apertura que permite su aplicación en contextos muy diversos. El servicio, planteado como una respuesta a necesidades reales de la sociedad y combinado con el aprendizaje añade valor y transforma a ambos (Puig et al., 2006). Por otro lado, cualificación y responsabilidad social deben formar un binomio inseparable, por ello con esta actividad pretendemos contribuir a la formación profesionales 
altamente capacitados y a la vez dotados de principios éticos que guíen su futura práctica profesional.

El presente proyecto se llevó a cabo en cuatro fases que se detallan a continuación.

\section{FASE 1: Búsqueda de información específica y desarrollo de materiales de aprendizaje por parte de los estudiantes de los tres grados involucrados.}

Esta fase tuvo como objetivo fundamental, la adquisición de los conocimientos previos sobre la teoría celular necesarios para el aprovechamiento de las actividades que se llevarán a cabo durante el proyecto. Las herramientas metodológicas básicas fueron el aprendizaje autónomo y el trabajo cooperativo. Además, se emplearon metodologías de aprendizaje basado en problemas. Las tareas concretas que llevaron a cabo los estudiantes en esta fase fueron:

Tarea 1.1 Diseño y elaboración de un muro dinámico con la herramienta padlet sobre "teoría celular" (Estudiantes de Farmacia). La herramienta PADLET permite crear muros virtuales y dinámicos en los que se puede organizar información de todo tipo (texto, imágenes, audio, video, esquemas). Los estudiantes de Farmacia en grupos diseñaron un muro con información técnica y científica fidedigna sobre la teoría celular.

Tarea 1.2: Práctica de laboratorio "Visualización de células de la mucosa oral" (Estudiantes de Farmacia). En grupos de 4 alumnos desarrollaron la práctica de microscopía en el laboratorio. Cada estudiante pudo obtener, observar y analizar las características de sus propias células. Posteriormente, deberían transmitir los conocimientos y destrezas adquiridos en la práctica a los futuros maestros. Por ello, tuvieron que identificar los principales puntos críticos en el desarrollo y comprensión de los conceptos abordados en la misma de cara a su transposición a cualquier tipo de interlocutor.

Tarea 1.3: Búsqueda bibliográfica y elaboración de un glosario sobre células (Estudiantes de Educación). De manera individual y no pautada, tuvieron que buscar información sobre la célula y elaborar un listado con todos los conceptos importantes para describirla y formular preguntas sobre aquellos que consideren especialmente complejos para su transmisión a los niños.

Tarea 1.4: Identificación de recursos científicos divulgativos (Estudiantes de Educación). Los estudiantes debían identificar recursos científicos para la elaboración de materiales científicos adaptados y manipulativos para su uso en el aula de primaria.

\section{FASE 2: Transposición didáctica}

Durante esta fase se fomentó la colaboración transdisciplinar entre estudiantes. Para ello se diseñó un seminario cooperativo en el que los estudiantes de los grados involucrados tuvieron que colaborar de forma activa y no jerarquizada para la consecución eficaz de los objetivos de aprendizaje y de servicio a la comunidad. Las actividades planificadas se corresponden con las tareas 2.1 y 2.2 que se detallan a continuación:

Tarea 2.1: Seminario cooperativo: "Descubramos juntos la célula" (Estudiantes de los Grados en Educación y Farmacia): Se llevó a cabo un seminario en el que todos los estudiantes trabajaron de forma conjunta. Se crearon grupos mixtos de estudiantes de modo que a lo largo 
de una hora y media trabajaram bajo un objetivo común: adaptar una práctica de microscopía compleja de nivel universitario para realizar un taller científico con niños de primaria a la vez que se adquieren destrezas de comunicación básicas para la divulgación científica. Con este taller se buscó un potente feedback entre estudiantes ayudando a todos ellos en la consecución de sus objetivos de aprendizaje. Los estudiantes de educación y periodismo recibirán formación específica. Esta formación permitirá profundizar en el concepto de célula a la vez que ayuda a los estudiantes de farmacia a adaptar sus conocimientos científicos complejos a un lenguaje coloquial comprensible para cualquier paciente.

Tarea 2.3: Taller práctico de microscopía celular (Estudiantes de ambos grados)

Tras el seminario cooperativo se llevó a cabo un taller en el que los estudiantes de farmacia junto con las docentes de este grado contribuyeron a la capacitación como divulgadores científicos de los estudiantes de Educación. El objetivo general fue la familiarización con el método científico de los estudiantes de educación a la vez que se desarrolla la adquisición de competencias fundamentales de comunicación para el futuro profesional de la salud.

En este taller todos los participantes tuvieron la posibilidad de realizar su propio "experimento científico" cuyo objetivo final fue la observación de células propias de su mucosa oral. Con el fin de garantizar la adquisición de los aprendizajes al final de la fase 2 los estudiantes debieron realizar los siguientes entregables:

Tarea 2.3.1 Diseño de un vídeo divulgativo (Estudiantes de Farmacia).

A partir de la información obtenida sobre necesidades formativas de sus compañeros con otra especialización académica, cada grupo realizó un vídeo minuto destinado a docentes de primaria o secundaria como material de referencia para explicar el concepto "Célula".

Tare 2.3.3: Elaboración de materiales didácticos manipulativos y adaptación del taller práctico de microscopía para niños (Estudiantes de educación)

Los estudiantes de Educación, una vez finalizada la tarea 2.1, debían diseñar y construir por parejas una maqueta manipulativa de la célula eucariota que pueda ser utilizada en el aula y que permita trabajar de manera intuitiva y amena conceptos relacionados con la célula. Además debieron adaptar el procedimiento y los materiales (protocolo de laboratorio) utilizados en el taller de laboratorio para su realización en un aula de primaria.

\section{FASE 3: Puesta en marcha del servicio "Conoce tus células"}

Los estudiantes de los dos grados participaron en una serie de talleres que se realizaron en el colegio Marie Curie de Zaragoza. En estos talleres se trabajaron en colaboración estrecha con los docentes y educadores del centro con el fin de adaptarlos a las necesidades concretas de los receptores de los mismos (Figuras 2 y 3 ).

\section{FASE 4: Evaluación, análisis y difusión de resultados}

Durante esta fase se pretende valorar la idoneidad de las herramientas de enseñanzaaprendizaje utilizadas para la consecución de los objetivos del proyecto. Con este fin, se llevaron a cabo una serie de actividades de evaluación durante las fases 1 a 4 cuyo objetivo fue determinar el impacto en discentes y docentes mediante diversas metodologías de 
evaluación cuantitativa y cualitativa (cuestionarios, encuesta de satisfacción, focus group). Dichas herramientas tratarán de inducir la reflexión sobre el propio proceso de enseñanza aprendizaje (metacognición) y la influencia que los conocimientos y destrezas de otros tienen sobre este (cooperación y sinergia entre iguales).

\section{Resultados}

Los estudiantes de farmacia obtuvieron calificaciones promedio de $6.8 \pm 1.6$ para el muro y 6,6 \pm 1.3 para el vídeo minuto. Los estudiantes de educación obtuvieron calificaciones promedio comprendidas entre 8.7 y 9.2 en las tareas evaluables. En ambos grados, los trabajos mostraron un alto nivel de compromiso, creatividad y entusiasmo. Además todos ellos una vez revisados, pudieron ser utilizados en los talleres divulgativos debido a su utilidad. En las figuras pueden observarse algunos de estos trabajos.

Tras la identificación de los mejores trabajos, se ofreció la posibilidad de participar de forma voluntaria en los talleres a los estudiantes de ambos grados. Se realizaron tres talleres de una hora de duración con los escolares de los tres grupos de $2^{\circ}$ de educación primaria del CEIP Marie Curie de Zaragoza (Figura 2). En cada taller participaron 25 escolares y 4 estudiantes Universitarios dos de cada Grado. Desde el grado en Farmacia se trasladaron 5 microscopios de docencia y todo el material de laboratorio necesario para realizar los talleres. Los niños fueron divididos en grupos de $5 \mathrm{y}$ asignados a un puesto de trabajo fueron guiados en todo momento por un estudiante universitario. Con el fin de garantizar que todos los procedimientos eran adecuados estuvieron presentes en todo momento la Maestra del grupo y la Docente de Fundamentos de Biología del grado en Farmacia. Al finalizar las sesiones los niños completaron un cuestionario sobre la actividad (Figura 3).

De los 70 niños participantes en los talleres, el 63\% nunca había observado a través de un microscopio por lo que la actividad resultó muy novedosa y estimulante. El 96,8\% de los niños participantes contestó que el taller les había gustado mucho y el 95,3\% que habían aprendido cosas que no sabían sobre las células. Además entre las valoraciones cualitativas que escribieron de la actividad destacan algunas como "Ha sido una experiencia super chula, me ha encantado mirar todas las células" "Por fín he visto mis células" "He aprendido mucho porque no sabía qué eran las células"

Respecto a los estudiantes participantes en el taller, destacaron como principal valor de la actividad la posibilidad de interacción con otros profesionales así como la oportunidad de realizar prácticas reales en entornos reales. Algunos comentarios recibidos por los docentes se destacan a continuación:

- "Ha sido una experiencia muy chula hemos aprendido un montón y lo hemos pasado fenomenal"

- "Le escribo para comunicarle mi total conformidad y gratitud por darme la oportunidad de participar en este proyecto"

- "Las actividades me encantaron y me resulto muy fácil desenvolverme por el aula". 


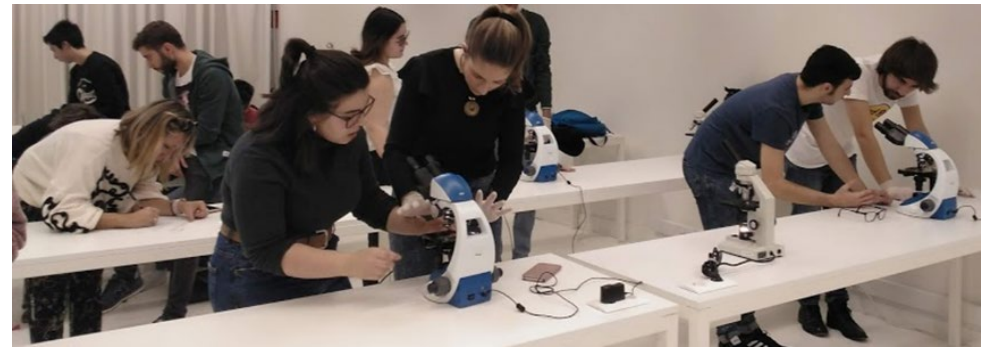

Fig1. Transposicón didáctica, taller cooperativo Intergrado (Farmacial Educación) Universidad San Jorge
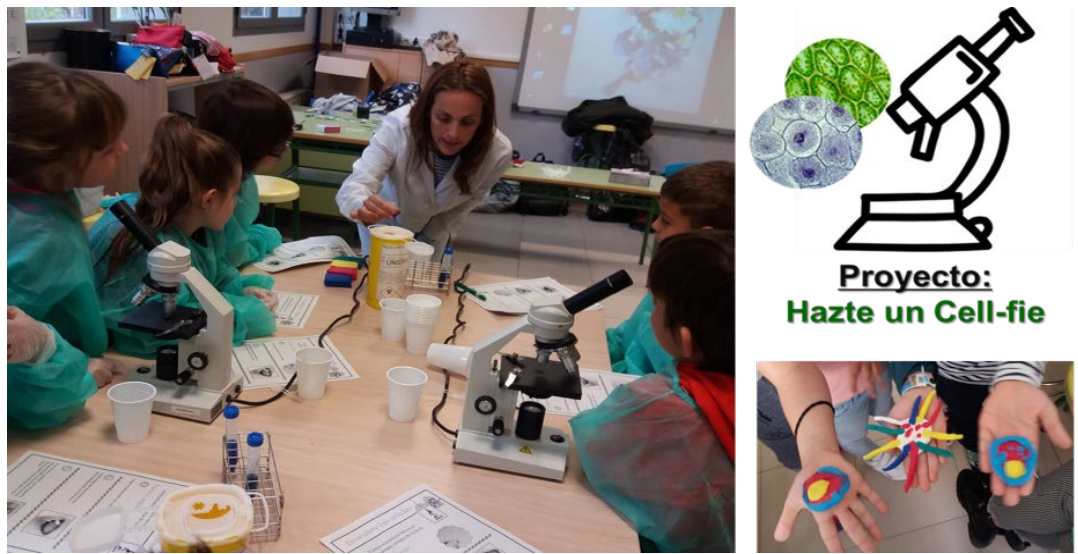

Fi 2. Talleres cientificos realizados en el colegio Marie Curie con alumnos de segundo de primaria y células de plastilina moldeadas por los escolares.
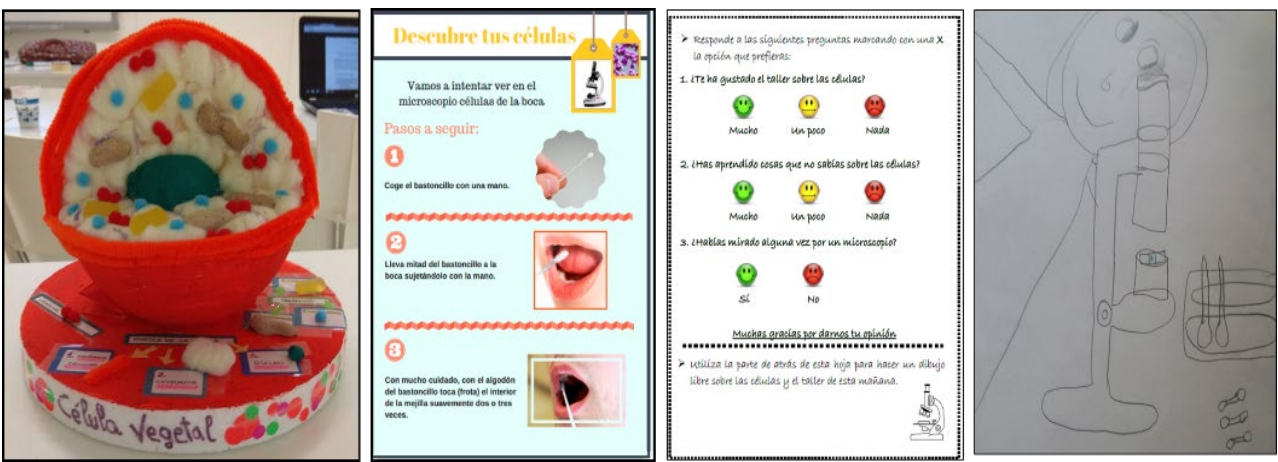

Fig 3. Maqueta de célula eucariota, protocolo de laboratorio adaptado para niños, cuestionario de la actividad, autorretrato realizado por una de las participantes. 


\section{Conclusiones}

Los resultados obtenidos en el presente trabajo pusieron de manifiesto la idoneidad de la metodología de Aprendizaje Servicio como estrategia para el fomento de la cultura científica entre escolares. Entre las actividades realizadas destaca la transposición didáctica entre estudiantes Universitarios de distintos grados dado ya que contribuye al conocimiento y valoración del trabajo de otros profesionales a la vez que contribuye a la difusión de la ciencia. Los resultados obtenidos en el proyecto "Hazte un Cell-fie" permiten asentar las bases para la realización anual de talleres científicos de distinta índole (biología, química, física, materiales). De este modo, la Universidad San Jorge asume el compromiso estable de contribuir y promover la "alfabetización científica" de distintos colectivos.

\section{Referencias}

- ABADÍA-VALLE, AR; MUÑOZ GONZALVO, MJ; SOTERAS, F. (2011). “¿Existen alternativas a las clases magistrales? Una experiencia en Fisiología Ocular del grado de Óptica y Optometría". Arbor, 187, 3, 189-194.

- EYLER, J, D. E. GILES, J. (1999). Where's the Learning in Service-Learning? San Francisco: Jossey-Bass.

- HONNET, E.P; POULSEN, S (1989). Principles of good practice in combining service and learning. Wingspread Special Report. Racine WI: The Johnson Foundation.

- LUCAS, S., Y MARTíNEZ-ODRÍA, A (2012). La implantación y difusión del Aprendizaje Servicio en el contexto educativo español: retos de futuro de una metodología de enseñanzaaprendizaje para promover la innovación en la Educación Superior En Libro de Actas del Congreso Internacional de Docencia Universitaria e Innovación. Universidad Pompeu Frabra, Barcelona. Recuperado de http://www.cidui.org/revistacidui12/index.php/cidui12/article/view/122.

- PALOS, J. (2009). ¿Por qué hacer actividades de Aprendizaje Servicio? En J.M. Puig (Coord.) Aprendizaje Servicio (ApS). Educación y compromiso cívico (pp.151-161). Barcelona: Graò.

-PUIG, JM ET AL (2006) Aprendizaje servicio. Educar para la ciudadanía. Editorial Octaedro.

-SABARIEGO, J,. Y MANZANARES, M. "Alfabetización científica" en l Congreso Iberoamericano de Ciencia, Tecnología, Sociedad e Innovación CTS + 1. Palacio de Minería. 2006.

- SOLVES, J. Y VIILCHES, A. (1997). "STS interactions and the teaching of physics and chemistry". Science Education, 81 (4), pp. 377-38.

-UNESCO-ICSU (1999-a) Declaración de Budapest sobre la Ciencia y el uso del saber científico. Conferencia Mundial sobre la Ciencia para el siglo XXI: Un nuevo compromiso, Budapest (Hungria), 26 junio - 1 julio de 1999.

-UNESCO-ICSU (1999b). Proyecto de programa en pro de la ciencia: Marco general de acción. Conferencia Mundial sobre la Ciencia para el siglo XXI: Un nuevo compromiso, Budapest (Hungria), 26 junio - 1 julio de 1999.

-WALTERS, MR. (1999). "Case-stimulated learning within endocrine physiology lectures: an approach applicable to other disciplines". Advance Physiology Education 276 , 74-78. 
Carlota Gómeza, Elisa Langaa , Jose Juan Verón; Marta Uriela , Diseré Acebes; Monika Wozniak, Eva $M^{a}$ Terrado

-Waters, J.R (2005). "Cat dissection vs. Sculpting human structures in clay: an analysis of two approaches to undergraduate human anatomy laboratory education". Advance Physiology Education 29,27-34. 